\title{
Ripening season, ethylene production and respiration rate are related to fruit non-destructively-analyzed volatiles measured by an electronic nose in 57 peach (Prunus persica L.) samples
}

\author{
Mingshen $\mathrm{Su}^{1 *}$, Zhengwen $\mathrm{Ye}^{1 *}$, Bo Zhang ${ }^{2}$, Kunsong Chen ${ }^{2}$ \\ ${ }^{1}$ Forestry and Pomology Research Institute, Shanghai Academy of Agricultural Sciences, Shanghai 201403, PR China, ${ }^{2}$ Laboratory of Fruit \\ Quality Biology, The State Agriculture Ministry Laboratory of Horticultural Plant Growth, Development and Quality Improvement, Zhejiang \\ University, Hangzhou 310058, PR China
}

${ }^{*}$ Co-first: Mingshen Su

\section{A B S T R A C T}

\begin{abstract}
Fifty-seven peach samples from 19 peach cultivars were gathered at consumption maturities from an orchard between July 21 and September 9 in 2010. Fruit ethylene production $\left(\mathrm{C}_{2} \mathrm{H}_{4}\right)$ was significantly positively correlated with respiration rate $\left(\mathrm{CO}_{2}\right)$ for tested samples. Fruit non-destructively-analyzed volatiles were measured by a FOX 4000 electronic nose (e-nose), which 57 samples were classified into three groups according to ripening season, respiration rate and ethylene production to evaluate the volatile separations. Samples harvested

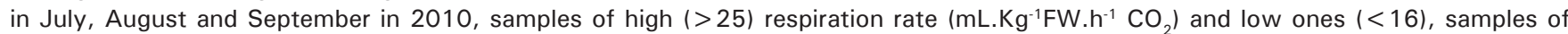
high $(>10)$ ethylene production $\left(n L . g^{-1} \mathrm{FW} \cdot \mathrm{h}^{-1} \mathrm{C}_{2} \mathrm{H}_{4}\right)$ and low ones $(<2)$ ones could be clearly separated in the discriminant factor analysis (DFA) plots based on fruit volatiles of the e-nose measurements. Intensity values of T30/1, T70/2, PA/2, P30/1, P40/2, P30/2 and T40/2 sensors of the e-nose measurements were in accord with the classifications of harvest seasons, respiration rate and ethylene production. non-destructively-analyzed volatiles, respiration rate and ethylene production were somewhat related each other despite genotype and environmental factor in the tested peach fruit samples.
\end{abstract}

Keywords: Peach fruit volatiles; non-destructively-analyzed characteristics; Three classifications; Separation relationships; Sensors

\section{INTRODUCTION}

Peach is one of the climacteric fruits which feature a stage of ethylene synthesis and respiration increase to produce edible fruits (Czarny et al., 2006). Ethylene is a naturally produced plant growth regulator. Both the synthesis and action of $\mathrm{C}_{2} \mathrm{H}_{4}$ are sensitive to concentrations of $\mathrm{CO}_{2}$ (Saltveit, 1999) $\mathrm{CO}_{2}$ regulates ethylene biosynthesis, at least in part, by counteracting ethylene action (Mathooko, 1996). Ethylene has significant effects on the quality of many harvested plant products. Ethylene alters volatiles and induces respiration in nearly all postharvest plant organs (Fugate et al., 2010).

However, the impact of ethylene on fruit volatile production is not yet well understood (Günthera et al.,
2011). Productions of volatiles and endogenous ethylene are not parallel in cut roses (Rosa bybrida L.). Exogenous ethylene has differential effects among cut rose varieties (Borda et al., 2011). Effects of MeJA on productions of fruit ethylene and volatiles differ among apple varieties (Kondo et al., 2005). Some differences of ethylenedependent and independent volatile synthesis exit between melon and apple fruits (Pech et al., 2008).

An electronic nose system considers the total headspace volatiles and creates a unique smell print (Zhang et al., 2008). E-noses are expected to give important indications in terms of the quality of the products and their marketable value (Natale et al., 2002). E-noses are instruments mimicking smell sense to detect and distinguish volatiles

\footnotetext{
*Corresponding authors:

Mingshen Su and Zhengwen Ye, Jinqi road 1000 of Fengxian district of Shanghai, Tel: 86-18918162163, Fax: 86-021-37195702, Korea.

E-mail: sumingshen2010@163.com and yezhengwen1300@163.com
}

Received: 18 January 2017; Revised: 01 October 2017; Accepted: 15 October 2017; Published Online: 21 October 2017 
in complex samples and widely applied in food control in this new century (Perisa and Escuder-gilabert, 2009). E-noses are used to monitor food quality based on volatiles (Wilson, 2013). E-noses could discriminate different rice (Oryza Sativa) genotypes (Zheng et al., 2009; Han et al., 2016), and different doenjang samples (Hong et al., 2015).

E-noses give viability to fruit distinguishing and grading quality in business markets. E-nose separations of aroma are separated by the volatiles present and molar proportions of individual segments found in every aroma (Baietto and Wilson, 2015). Fruit volatiles of four peach cultivars at different stages of room temperature storage and of $0^{\circ} \mathrm{C}$ storage could be distinguished by an e-nose (Benedetti et al., 2008; Infante et al., 2008). Fruit volatiles of 'Spring Belle' peach of different cold storage time at 0 and $4^{\circ} \mathrm{C}$ could be discriminated by an e-nose (Rizzolo et al., 2013). Peach and nectarine pulp samples at harvest of low TSS concentration and TSS/TA ratio could be differentiated from high TSS concentration and TSS/TA ratio in DFA plots of based on pulp volatiles measured by an e-nose despite genotypes, respectively (Su et al., 2013). However, it is still indispensable to further explore relationships of non-destructively-analyzed volatiles measured by an e-nose and fruit characteristics in peach samples.

In this study, ethylene production, respiration rate and e-nose measured volatiles were investigated in 57 fruit samples from 19 peach cultivars at harvest level, and the outline relationships between each other were evaluated despite genotypes and environmental factors.

\section{MATERIALS AND METHODS}

\section{Experimental material}

57 fruit samples from 19 peach cultivars were gathered from a peach germplasm orchard in Shanghai academy of agricultural sciences, China. Fruit of each cultivar were harvested between July 21 and September 9 in 2010 at commercial maturity.

Three or two fruit of each cultivar were mixed together as one replicate, and three replicates of each cultivar were measured to compose of 57 fruit samples. Each fruit sample was sealed in a $2 \mathrm{~L}$ flask at $20 \pm 1^{\circ} \mathrm{C}$ for $1 \mathrm{~h}$, then $1 \mathrm{~mL}$ of headspace gas was collected to measure respiration rate, ethylene production and non-destructively-analyzed volatiles, respectively.

\section{Respiration rate and ethylene production measurements} For respiration rate, $1 \mathrm{~mL}$ of gas sample was injected into an infra-red equipment of ICA 40 (International Controlled Atmosphere Ltd., UK) to detect CO2 concentration according to the method of Zhang et al., (2005).
Ethylene production was assayed according to the method of Zhang et al., (2005) with slight modifications. $1 \mathrm{~mL}$ of gas sample was injected into a gas chromatograph of model SP 6800 (Lunan Chemical Engineering Instrument Co. Ltd., Shandong, China) using a detector fitted with a GDX-502 column (Shanghai Hui Fen Scientific Analysis Instrument Co. Ltd., Shanghai, China). The N2 carrier gas was at $0.18 \mathrm{mPa}$, while air gas and $\mathrm{H} 2$ gas in the flame ionization detector (FID) was 0.08 and $0.05 \mathrm{mPa}$, respectively. The temperatures of injector, detector and oven were controlled at 110 , 140 and $90{ }^{\circ} \mathrm{C}$, respectively.

\section{Electronic nose analysis}

The non-destructively-analyzed electronic nose measurement was assayed according to the method of Lebrun et al., (2008) with modifications. The e-nose FOX 4000 (Alpha MOS, Toulouse, France) equipped with an automatic headspace sampler HS100 (Alpha MOS) and 18 metallic oxide sensors was used to analyze fruit volatiles. The 18 sensors are named LY2/LG, LY2/G, LY2/AA, LY2/GH, LY2/gCTl, LY2/gCT, T30/1, P10/1, P10/2, P40/1, T70/2, PA/2, P30/1, P40/2, P30/2, T40/2, T40/1, and TA/2. There are LY2, $\mathrm{P}$ and $\mathrm{T}$ three types of metal oxide sensors, which LY2 sensors are based on chromium titanium oxide $\left(\mathrm{Cr}_{2}-\mathrm{xTixO}_{3}+\mathrm{y}\right)$ and on tungsten oxide $\left(\mathrm{WO}_{3}\right)$, while $\mathrm{P}$ and $\mathrm{T}$ sensors are based on tin dioxide $\left(\mathrm{SnO}_{2}\right)$ placed on a plain substrate and a aluminum tube, respectively (Huang et al., 2015).

$1 \mathrm{~mL}$ of headspace gas sampled with a syringe was injected into the equipment. The headspace gas was pumped into the sensor chamber with a constant rate of $100 \mathrm{~mL} / \mathrm{min}$ via a Teflon-tubing connected to a needle during the measurement process. The measurement phase of signal acquisition lasted for $120 \mathrm{~s}$, and the clean phase of baseline recovery lasted for $240 \mathrm{~s}$.

\section{Data processing}

Data were presented as mean values \pm standard error (SE). Data from the electronic nose were analyzed using discriminant factor analysis (DFA) to observe the relevant features followed fruit characteristics. DFA were determined applying the statistical program Alpha Soft Version 11.0 according to the method of Huang et al., (2011).

\section{RESULTS}

\section{Fruit characteristics and classifications of tested 57} peach samples

57 fruit samples from 19 peach cultivars in which 3 samples for each cultivar were collected in the study, which ripening date ranged from July 21 until September 9, lasted for $50 \mathrm{~d}$. 
Ethylene production of fruit samples ranged from 0.56 to $15.26 \mathrm{~nL} \mathrm{~g}^{-1} \mathrm{FW} \mathrm{h}{ }^{-1}$, the highest one accounted 27.25 times for the lowest one. Respiration rate of fruit samples ranged from 13.60 to $39.20 \mathrm{~mL} \mathrm{Kg}^{-1} \mathrm{FW} \mathrm{h}{ }^{-1}$, the highest one accounted 2.88 times for the lowest one. Variation of ethylene production was higher than respiration rate in the tested 57 fruit samples (Table 1).

Ethylene production and respiration rate of fruit samples ripened in August were highest, followed by those in July, and those in September were lowest (Fig. 1A and 1B). Significant positive linear relationships were observed between respiration rate and ethylene production in tested 57 samples, $\mathrm{R}$ reached 0.6509 ( $\mathrm{P}<0.01$ ) (Fig. 1C).

Fruit samples were classified into three groups termed group A, B and C to evaluate separations of nondestructively-analyzed volatiles measured by the e-nose in the later study. Fruit samples ripened in July, August and September were classified into A, B and C groups, respectively. Fruit samples of ethylene production $<2,2-10$ and $>10$ nL.g-1 FW.h-1 were classified into A, B and C groups, respectively. Fruit samples of respiration rate $<16$, 16-25 and $>25$ mL.Kg-1FW.h-1 were classified into A, B and $\mathrm{C}$ groups, respectively (Table 2 ).

Separations of classified fruit samples in the DFA (discriminant factor analysis) plots based on nondestructively-analyzed volatiles of the e-nose measurements

The DF1 and DF2 accounted for $68.997 \%$ and $31.003 \%$ of the total DFA variance in the DFA plot of 57 fruit samples of three ripening seasons, respectively. Samples ripened in July, August and September could be clearly clustered into three independent groups in the DFA plot. The separations were mainly achieved along the DF1 axis, which samples ripened in July located on the left side, and those in September located on the right side (Fig. 2).
The DF1 and DF2 accounted for $80.760 \%$ and $19.240 \%$ of the total DFA variance in the plot of 57 samples of three ethylene production levels, respectively. Samples of high ethylene production could be clearly distinguished from low ones, while medium ones located on the position between low and high ones along the DF1 axis in the DFA plot (Fig. 3A). The DF1 and DF2 accounted for $79.222 \%$ and $20.778 \%$ of the total DFA variance in the plot of 57 fruit samples of three respiration rate levels, respectively. Samples of high levels could be clearly distinguished from low ones, while medium ones overlapped with low and high ones in the DFA plot (Fig. 3B).

Intensity values of 18 sensors in response to three classifications of tested samples based on fruit volatiles of e-nose measurements

Intensity values of LY2/LG, LY2/G, LY2/AA, LY2/GH, $\mathrm{LY} 2 / \mathrm{gCT} 1$ and LY2/gCT sensors were tiny for all tested samples, in which LY2/G, LY2/AA, LY2/GH, LY2/gCT1 and LY2/gCT sensors were negative (Fig. 4A-C). Intensity values of T30/1, T70/2, PA/2, P30/1, P40/2, P30/2 and $\mathrm{T} 40 / 2$ sensors for samples ripened in August (higher ethylene and respiration) (Fig. 4A), high ethylene (Fig. 4B) and high respiration (Fig. 4C) were highest, followed by those in July (moderate ethylene and respiration) (Fig. 4A), medium ethylene (Fig. 4B) and medium respiration (Fig. 4C), and those in September (lower ethylene and respiration) (Fig. 4A), low ethylene (Fig. 4B) and low respiration (Fig. 4C) were lowest, respectively. Intensity values of $\mathrm{P} 10 / 1, \mathrm{P} 10 / 2, \mathrm{P} 40 / 1, \mathrm{~T} 40 / 1$ and TA/2 sensors did not coincided exactly with three classifications of both ethylene and respiration in tested samples based on fruit volatiles of e-nose measurements (Fig. 4A -C).

\section{DISCUSSION}

Exogenous ethylene can increase respiration rate in sugar beet (Beta vulgaris L.) roots (Fugate et al., 2010). Respiration

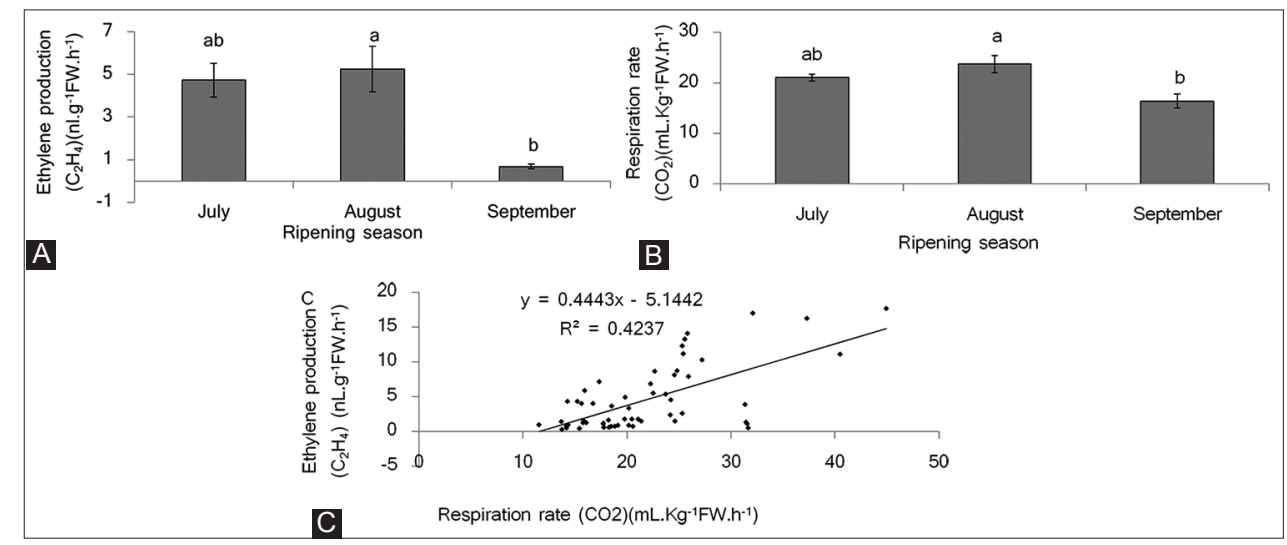

Fig 1. (A) Ethylene production, (B) Respiration rate and (C) linear relationships between ethylene production and respiration rate of the tested 57 samples ripened in July, August and September, respectively. 
$\mathrm{Su}$, et al.:Peach e-nose volatiles are related to $\mathrm{C}_{2} \mathrm{H}_{4}$ and $\mathrm{CO}_{2}$

Table 1: Peach cultivars, ripening date, ethylene production and respiration rate of $\mathbf{5 7}$ fruit samples

\begin{tabular}{|c|c|c|c|c|}
\hline Peach cultivars & Ripening date & Fruit samples & $\begin{array}{l}\text { Ethylene production }\left(\mathrm{C}_{2} \mathrm{H}_{4}\right) \\
\left(\mathrm{nL} \cdot \mathrm{g}^{-1} \mathrm{FW} \cdot \mathrm{h}^{-1}\right)\end{array}$ & $\begin{array}{l}\text { Respiration rate }\left(\mathrm{CO}_{2}\right) \\
\left(\mathrm{mL} \cdot \mathrm{Kg}^{-1} \mathrm{FW} \cdot \mathrm{h}^{-1}\right)\end{array}$ \\
\hline \multirow[t]{3}{*}{ Qianqu } & Jul. 21 & 1 & 1.47 & 24.63 \\
\hline & & 2 & 2.42 & 24.20 \\
\hline & & 3 & 4.54 & 24.23 \\
\hline \multirow[t]{3}{*}{ Dajiubao } & Jul. 21 & 1 & 1.79 & 19.77 \\
\hline & & 2 & 0.77 & 20.58 \\
\hline & & 3 & 0.59 & 17.78 \\
\hline \multirow[t]{3}{*}{ Xingfeng } & Jul. 21 & 1 & 1.78 & 21.09 \\
\hline & & 2 & 1.21 & 17.73 \\
\hline & & 3 & 6.84 & 22.30 \\
\hline \multirow[t]{3}{*}{ Momu } & Jul. 21 & 1 & 1.14 & 17.73 \\
\hline & & 2 & 0.71 & 18.51 \\
\hline & & 3 & 1.65 & 18.26 \\
\hline \multirow[t]{3}{*}{ Dazhenbao } & Jul. 27 & 1 & 8.74 & 24.84 \\
\hline & & 2 & 3.68 & 18.53 \\
\hline & & 3 & 5.39 & 23.72 \\
\hline \multirow[t]{3}{*}{ Zhongjin } & Jul. 27 & 1 & 7.16 & 17.33 \\
\hline & & 2 & 8.67 & 22.67 \\
\hline & & 3 & 5.91 & 15.94 \\
\hline \multirow[t]{3}{*}{ YunshuNo. 1} & Jul. 27 & 1 & 10.26 & 27.24 \\
\hline & & 2 & 14.06 & 25.81 \\
\hline & & 3 & 12.31 & 25.32 \\
\hline \multirow[t]{3}{*}{ Zaobaihua } & Jul. 27 & 1 & 3.36 & 20.18 \\
\hline & & 2 & 4.95 & 19.85 \\
\hline & & 3 & 4.03 & 16.73 \\
\hline \multirow[t]{3}{*}{ Tanghang } & Aug. 1 & 1 & 4.33 & 14.29 \\
\hline & & 2 & 4.29 & 15.24 \\
\hline & & 3 & 4.06 & 15.67 \\
\hline \multirow[t]{3}{*}{ Cuanzhongdao } & Aug. 1 & 1 & 0.51 & 14.18 \\
\hline & & 2 & 1.44 & 13.69 \\
\hline & & 3 & 1.58 & 15.84 \\
\hline \multirow[t]{3}{*}{ Hu463 } & Aug. 1 & 1 & 1.28 & 16.08 \\
\hline & & 2 & 1.49 & 21.41 \\
\hline & & 3 & 0.74 & 18.84 \\
\hline \multirow[t]{3}{*}{ Qingshui } & Aug. 4 & 1 & 17.00 & 32.11 \\
\hline & & 2 & 17.69 & 44.92 \\
\hline & & 3 & 11.09 & 40.48 \\
\hline \multirow[t]{3}{*}{ Baihua } & Aug. 4 & 1 & 5.53 & 22.54 \\
\hline & & 2 & 8.09 & 24.60 \\
\hline & & 3 & 1.76 & 20.47 \\
\hline \multirow[t]{3}{*}{ Jinyuan } & Aug. 13 & 1 & 7.88 & 25.92 \\
\hline & & 2 & 2.61 & 25.35 \\
\hline & & 3 & 3.91 & 31.36 \\
\hline \multirow[t]{3}{*}{ Qiuyue } & Aug. 13 & 1 & 16.22 & 37.31 \\
\hline & & 2 & 11.19 & 25.44 \\
\hline & & 3 & 13.25 & 25.59 \\
\hline \multirow[t]{3}{*}{ Jinxiu } & Aug. 17 & 1 & 1.23 & 15.81 \\
\hline & & 2 & 0.96 & 14.36 \\
\hline & & 3 & 0.79 & 14.20 \\
\hline \multirow[t]{3}{*}{ Ergong } & Aug. 17 & 1 & 1.33 & 31.48 \\
\hline & & 2 & 1.09 & 31.56 \\
\hline & & 3 & 0.49 & 31.69 \\
\hline \multirow[t]{3}{*}{ Jinhua } & Sep. 9 & 1 & 0.92 & 19.12 \\
\hline & & 2 & 0.91 & 20.21 \\
\hline & & 3 & 0.59 & 18.29 \\
\hline Jinshuo & Sep. 9 & 1 & 0.42 & 15.47 \\
\hline & & 2 & 0.30 & 13.76 \\
\hline & & 3 & 0.96 & 11.57 \\
\hline
\end{tabular}


rate is elevated with ethylene levels in nectarine fruits removed from chilling temperatures (Zhou et al., 2001). Significant positive linear relationships were existed between respiration rate and ethylene production in tested 57 peach samples in our study (Fig. 1C), it suggested that respiration rate was somewhat cooperated with ethylene production in peaches despite genotype and environmental factor.
Using DFA, fruit volatiles of the e-nose measurements could be separated according to fruit ripening seasons among 57 peach samples in our study (Fig. 2). Similarly somewhat, pulp volatiles measured by headspace solid phase micro extraction (HS-SPME) followed by gas chromatography/mass spectrometry (GC/MS) are related to fruit harvest seasons in apricot (Prunus armeniaca L.) samples (Bianco et al., 2010). Pulp volatiles of measured by

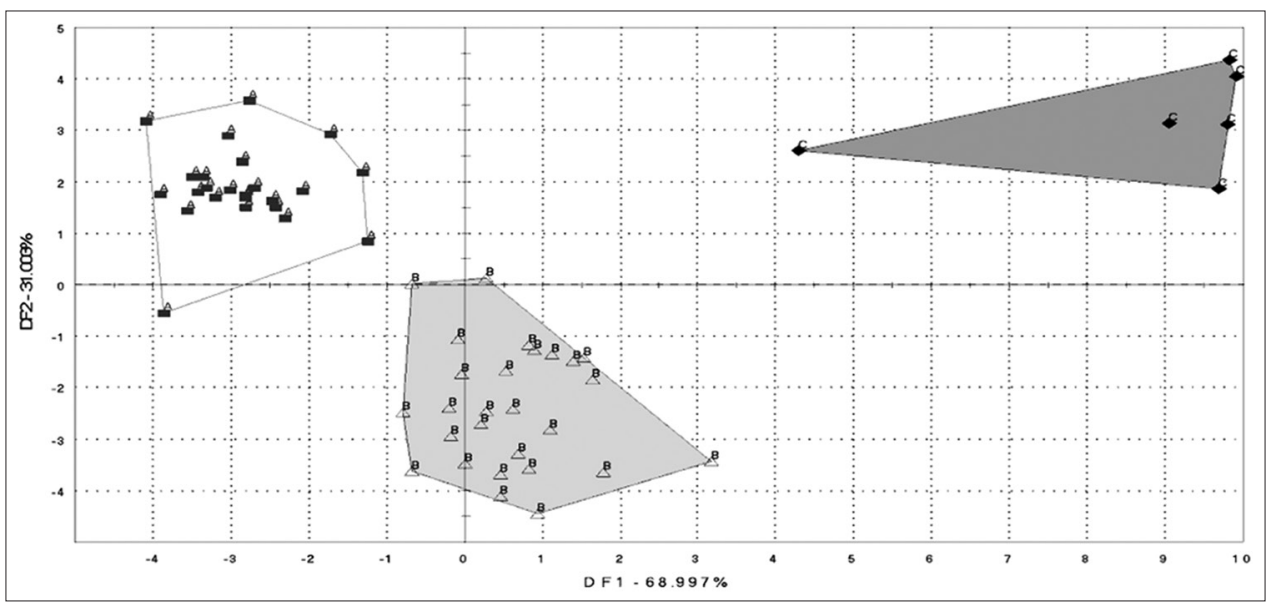

Fig 2. DFA plot of fruit samples ripened in July, August and September represented in white background A background $C \diamond$ based on volatiles of the e-nose measurements, respectively.

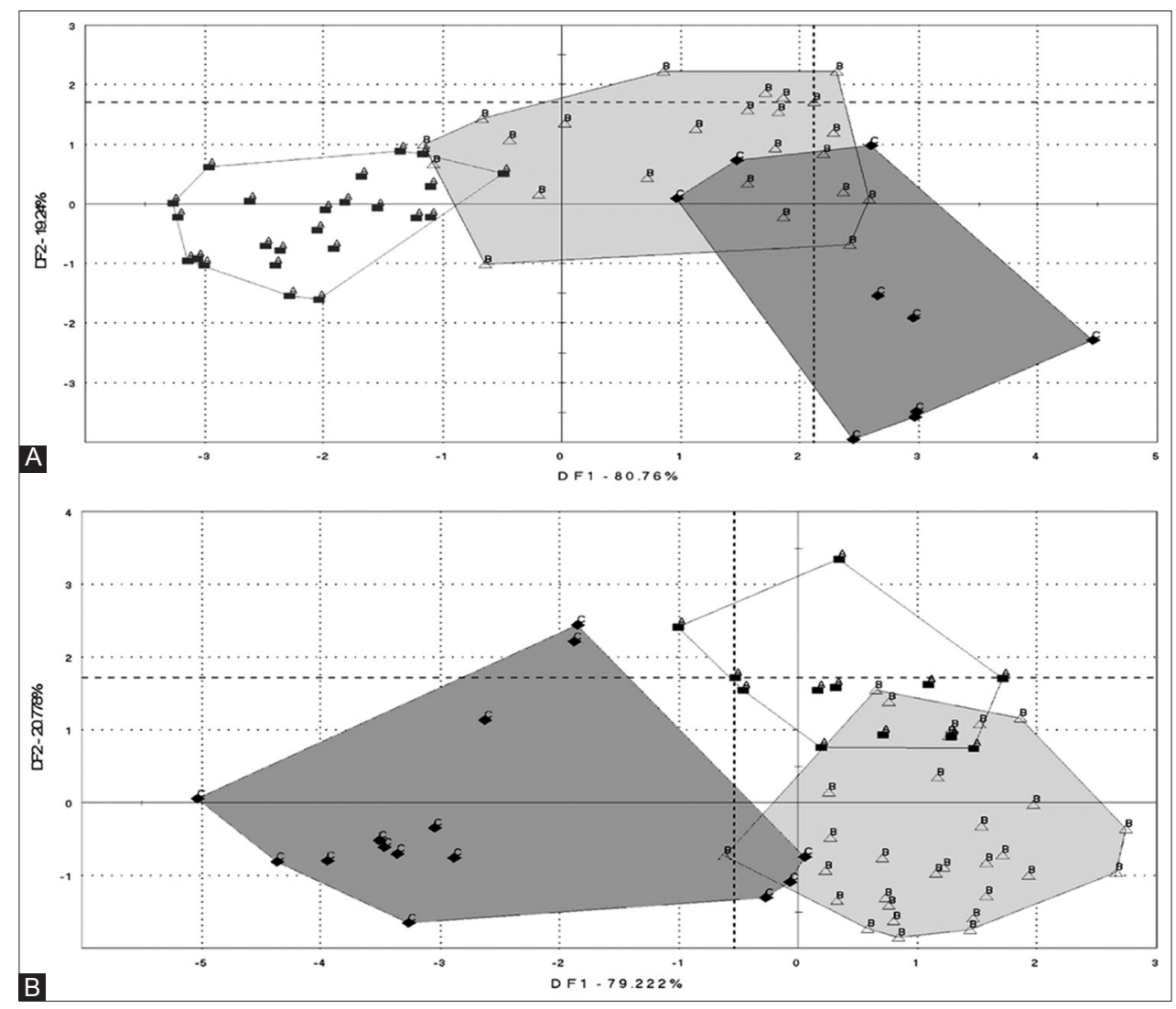

Fig 3. The DFA plots of fruit samples classified by ethylene production (A) and respiration rate $(B)$ in low, medium and high levels represented in white background $A \boldsymbol{A}$, light background $B \boldsymbol{\Delta}$ and dark background $C \wedge$ based on volatiles of the e-nose measurements, respectively. 


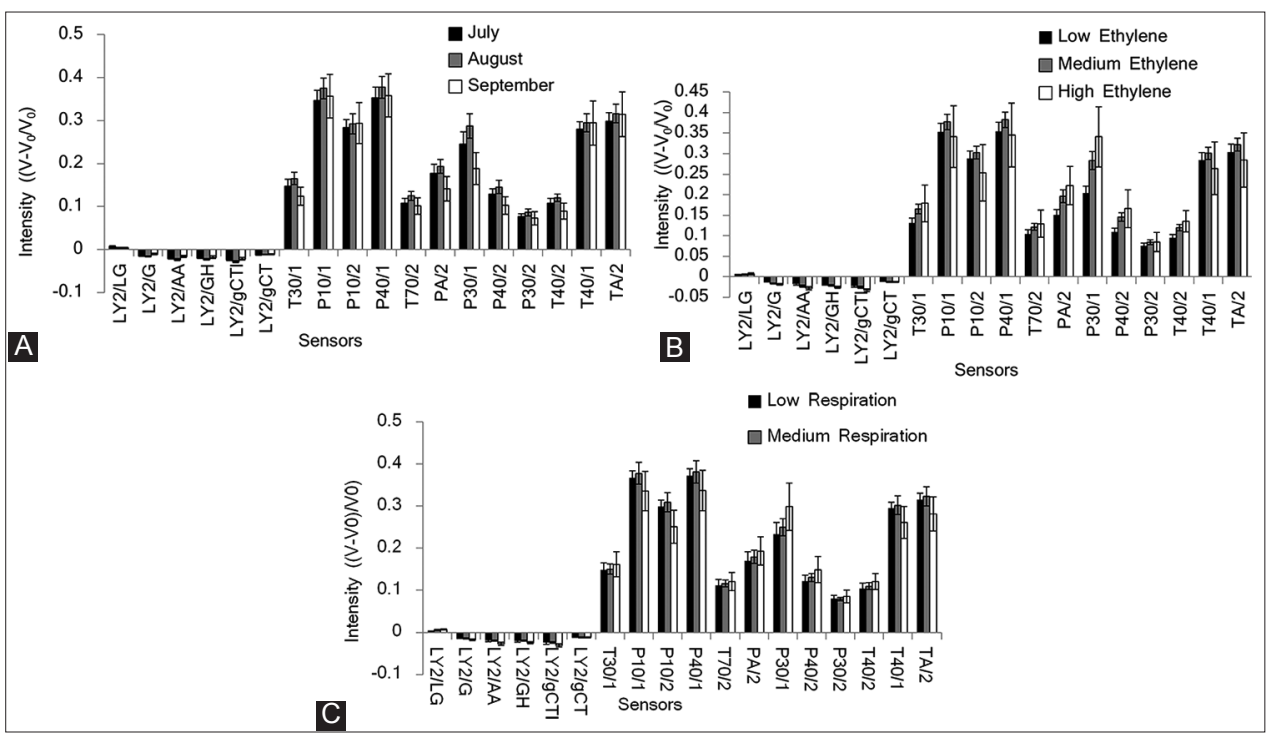

Fig 4. Intensity values of 18 sensors in response to three classifications of (A) ripening seasons, $(B)$ respiration rate and (C) ethylene production in tested samples based on fruit volatiles of e-nose measurements, respectively

Table 2: Standards and numbers of fruit samples according to three classifications of ripening season, ethylene production and respiration rate for the tested 57 samples, respectively

\begin{tabular}{|c|c|c|c|c|c|c|}
\hline \multirow[t]{2}{*}{ Classification } & \multicolumn{2}{|c|}{ Sample A } & \multicolumn{2}{|c|}{ Sample B } & \multicolumn{2}{|c|}{ Sample C } \\
\hline & Standard & Number & Standard & Number & Standard & Number \\
\hline Ripening season & July & 24 & August & 27 & September & 6 \\
\hline Ethylene production $\left(\mathrm{C}_{2} \mathrm{H}_{4}\right)\left(\mathrm{nL} \cdot \mathrm{g}^{-1} \mathrm{FW} \cdot \mathrm{h}^{-1}\right)$ & $<2$ (low) & 24 & 2-10 (medium) & 24 & $>10$ (high) & 9 \\
\hline Respiration rate $\left(\mathrm{CO}_{2}\right)\left(\mathrm{mL} . \mathrm{Kg}^{-1} \mathrm{FW} \cdot \mathrm{h}^{-1}\right)$ & $<16$ (low) & 12 & $16-25$ (medium) & 30 & $>25$ (high) & 15 \\
\hline
\end{tabular}

an e-nose are related to fruit harvest seasons in peach and nectarines samples (Su et al., 2013). These results suggested that somewhat relationships between fruit whole volatiles and harvest season were also existed in peach samples despite genotype and environmental factor.

Fruit volatile production is closely related to the changes of ethylene production in apple (Song and Bangerth, 1996) and peach (Zhang et al., 2010). Biosynthesis of monoterpenes, esters and aldehydes in the mango fruit is strongly dependent on ethylene production and action (Lalel et al., 2003). Most esters identified in mountain papaya (Vasconcellea pubescens) are affected by ethylene (Balbontìn et al., 2007). High correlations between ethylene production and aldehydes and alcohols contents are found in'Golden Delicious'apple (Salas et al., 2011). $\mathrm{CO}_{2}$ levels are important in determining the resultant production of volatiles (Toivonen, 1997). Volatiles are closely related to the changes in the respiration rate in 'Golden Delicious' apple fruit (Song and Bangerth, 1996). In our study, the e-nose could distinguish fruit samples of high ethylene production and respiration rate from low ones using DFA, respectively (Fig. 3A and 3B). Similarly, peach pulp samples of high TSS concentration and TSS/TA ratio could be distinguished from low ones based on pulp volatiles measured by an e-nose using DFA, respectively (Su et al., 2013). These results suggested that somewhat relationships between fruit whole non-destructively-analyzed volatiles and both ethylene production and respiration rate were existed in peaches despite genotype and environmental factor.

E-noses have non-linearity characteristics of sensor response to the odors called "ffingerprint" (Yan et al., 2015). In our study, intensity values of T30/1, T70/2, $\mathrm{PA} / 2, \mathrm{P} 30 / 1, \mathrm{P} 40 / 2, \mathrm{P} 30 / 2$ and $\mathrm{T} 40 / 2$ sensors were in accord with all the three classifications of harvest season (ethylene and respiration, too), respiration rate and ethylene production (Fig. 4A-C), these results suggested that these seven $\mathrm{P} \& \mathrm{~T}$ sensors contributed more important roles compared to the other 11 sensors in differentiating fruit non-destructively-analyzed volatiles of peach samples. Intensity values of LY2/LG, LY2/G, LY2/AA, LY2/GH, LY2/gCT1 and LY2/gCT sensors were tiny (Fig. 4A-C), these results suggested that LY2 sensors played little roles in differentiating fruit non-destructively-analyzed volatiles of peach samples. Possibly, it is worthy of paying more attention to T30/1, T70/2, PA/2, P30/1, P40/2, P30/2 and $\mathrm{T} 40 / 2$ sensors for further study in peach volatiles of the e-nose measurements. 


\section{CONCLUSION}

Ethylene production was significantly positively correlated with respiration rate, and variation of ethylene production was higher than that of respiration rate in the tested 57 peach samples at harvest level. Samples harvested in July, August and September, samples of high $(>25)$ and low $(<16)$ respiration rate (mL.Kg-1FW.h-1 CO2), and samples of high $(>10)$ and low $(<2)$ ethylene production (nL.g-1 FW.h-1 C2H4) could be clearly distinguished in the DFA plots of the e-nose measurements, respectively. T30/1, T70/2, PA/2, P30/1, P40/2, P30/2 and T40/2 sensors of the e-nose played considerable roles in differentiating fruit non-destructively-analyzed volatiles of classified peach samples.

\section{ACKNOWLEDGMENTS}

This work was supported by national peach production system of agriculture ministry of China (CARS-31-3-05), Shanghai Science and Technology Project [(15391901400), (HuNong2012No.1-3)] and Shanghai fruit production system (HuNong2014No.7).

\section{Authors' contributions}

Mingshen Su wrote the manuscript, carried out the statistical analysis. Zhengwen Ye, Bo Zhang and Kunsong Chen were involved in overall planning and supervision of the experiments and edited the manuscript.

\section{REFERENCES}

Baietto, M. and A. D. Wilson. 2015. Electronic-nose applications for fruit identification, ripeness and quality grading. Sensors. 15: 899-931.

Balbontìn, C., C. Gaete-Eastman, M. Vergara, R. Herrera and M. A. Moya-León. 2007. Treatment with 1-MCP and the role of ethylene in aroma development of mountain papaya fruit. Postharvest Biol Technol. 43: 67-77.

Benedetti, S., S. Buratti, A. Spinardi, S. Mannino and I. Mignani. 2008. Electronic nose as a non-destructive tool to characterize peach cultivars and to monitor their ripening stage during shelflife. Postharvest Biol Technol. 47: 181-188.

Bianco, R. L., V. Farina, S. G. Indelicato, F. Filizzola and P. Agozzino. 2010. Fruit physical, chemical and aromatic attributes of early, intermediate and late apricot cultivars. J. Sci. Food Agric. 90: 1008-1019.

Borda, A. M., D. G. Clark, D. J. Huber, B. A. Welt and T. A. Nell. 2011. Effects of ethylene on volatile emission and fragrance in cut roses: The relationship between fragrance and vase life. Postharvest Biol. Technol. 59: 245-252.

Czarny, J. C., V. P. Grichko and B. R. Glick. 2006. Genetic modulation of ethylene biosynthesis and signaling in plants. Biotechnol. Adv. 24: 410-419.

Fugate, K. K., J. C. Suttle and L. G. Campbell. 2010. Ethylene production and ethylene effects on respiration rate of postharvest sugarbeet roots. Postharvest Biol. Technol. 56: 71-76.
Günthera, C. S., K. Heinemanna, W. A. Laing, L. Nicolau and K. B. Marsh. 2011. Ethylene-regulated (methylsulfanyl) alkanoate ester biosynthesis is likely to be modulated by precursor availability in Actinidia chinensis genotypes. J Plant Physiol. 168: 629-638.

Han, H. J., S. H. Lee, J. Y. Moon, S. Park, H. Dong and B. S. Noh. 2016. Discrimination of the cultivar, growing region, and geographical origin of rice (s) using a mass spectrometer-based electronic nose. Food Sci. Biotechnol. 25: 695-700.

Hong, Y., B. S. Noh and H. Y. Kim. 2015. Discrimination of Doenjang samples using a mass spectrometry-based electronic nose and human sensory preference testing. Food Sci. Biotechnol. 24: 31-36.

Huang, L. X., H. R. Liu, B. Zhang and D. Wu. 2015. Application of electronic nose with multivariate analysis and sensor selection for botanical origin identification and quality determination of honey. Food Bioprocess Technol. 8: 359-370.

Huang, Y., F. Li, Y. P. Xia and K. S. Chen. 2011. Scent profiling of Cymbidium ensifolium by electronic nose. Sci. Hortic. 128: 306310.

Infante, R., M. Farcuh and C. Meneses. 2008. Monitoring the sensorial quality and aroma through an electronic nose in peaches during cold storage. J. Sci. Food Agric. 88: 2073-2078.

Kondo, S., S. Setha, D. R. Rudell, D. A. Buchanan and J. P. Mattheis. 2005. Aroma volatile biosynthesis in apples affected by 1-MCP and methyl jasmonate. Postharvest Biol. Technol. 36: 61-68.

Lalel, H. J. D., Z. Singh and S. C. Tan. 2003. The role of ethylene in mango fruit aroma volatiles biosynthesis. J. Hortic. Sci. Biotech. 78: 485-496.

Lebrun, M., A. Plotto, K. Goodner, M. N. Ducamp and E. Baldwin. 2008. Discrimination of mango fruit maturity by volatiles using the electronic nose and gas chromatography. Postharvest Biol. Technol. 48: 122-131.

Mathooko, F. M. 1996. Regulation of ethylene biosynthesis by carbon dioxide. Postharvest Biol. Technol. 7: 1-26.

Natale, C. D., M. Zude-Sasse, A. Macagnano, R. Paolesse, B. Herold and A. D. Amico. 2002. Outer product analysis of electronic nose and visible spectra: Application to the measurement of peach fruit characteristics. Anal. Chim. Acta. 459: 107-117.

Pech, J. C., M. Bouzayen and A. Latché. 2008. Climacteric fruit ripening: Ethylene-dependent and independent regulation of ripening pathways in melon fruit. Plant Sci. 175: 114-120.

Perisa, M. and L. Escuder-Gilabert. 2009. A 21st century technique for food control: Electronic noses. Anal. Chim. Acta. 638: 1-15.

Rizzolo, A., G. Bianchi, M. Vanoli, S. Lurie, L. Spinelli and A. Torricelli. 2013. Electronic nose to detect volatile compound profile and quality changes in 'spring belle' peach (Prunus persica L.) during cold storage in relation to fruit optical properties measured by time-resolved reflectance spectroscopy. J. Agric. Food Chem. 61: 1671-1685.

Salas, N. A., F. J. Molina-Corral, G. A. González-Aguilar, A. Otero, D. R. Sepulveda and G. I. Olivas. 2011. Volatile production by 'golden delicious' apples is affected by preharvest application of aminoethoxyvinylglycine. Sci. Hortic. 130: 436-444.

Saltveit, M. E. 1999. Effect of ethylene on quality of fresh fruits and vegetables. Postharvest Biol. Technol. 15: 279-292.

Song, J. and F. Bangerth. 1996. The effect of harvest date on aroma compound production from 'golden delicious' apple fruit and relationship to respiration and ethylene production. Postharvest Biol. Technol. 8: 259-269.

Su, M. S., B. Zhang, Z. W. Ye, K. S. Chen, J. Guo, X. J. Gu and J. Y. Shen. 2013. Pulp volatiles measured by an electronic nose 
are related to harvest season, TSS concentration and TSS/ TA ratio among 39 peaches and nectarines. Sci. Hortic. 150: 146-153.

Toivonen, P. M. A. 1997. Non-ethylene, non-respiratory volatiles in harvested fruits and vegetables: Their occurrence, biological activity and control. Postharvest Biol. Technol. 12: 109-125.

Wilson, A. D. 2013. Diverse applications of electronic-nose technologies in agriculture and forestry. Sensors. 13: 2295-2348.

Yan, J., X. Z. Guo, S. K. Duan, P. F. Jia, L. D. Wang, C. Peng and S. L. Zhang. 2015. Electronic nose feature extraction methods: A review. Sensors. 15: 27804-27831.

Zhang, B., J. Y. Shen, W. W. Wei, W. P. Xi, C. J. Xu, I. Ferguson and K. S. Chen. 2010. Expression of genes associated with aroma formation derived from the fatty acid pathway during peach fruit ripening. J. Agric. Food Chem. 58: 6157-6165.

Zhang, H. M., M. X. Chang, J. Wang and S. Ye. 2008. Evaluation of peach quality indices using an electronic nose by MLR, QPST and BP network. Sens. Actuators B Chem. 134: 332-338.

Zhang, W. S., K. S. Chen, B. Zhang, C. D. Sun, C. Cai, C. H. Zhou, W. P. Xu, W. Q. Zhang and I. Ferguson. 2005. Postharvest responses of Chinese bayberry fruit. Postharvest Biol. Technol. 37: 241-251.

Zheng, X. Z., Y. B. Lan, J. M. Zhu, J. Westbrook, W. C. Hoffmann and R. E. Lacey. 2009. Rapid identification of rice samples using an electronic nose. J. Bionic Eng. 6: 290-297.

Zhou, H. W., L. Dong, R. Ben-Arie and S. Lurie. 2001. The role of ethylene in the prevention of chilling injury in nectarines. J. Plant Physiol. 158: 55-61. 